\title{
Effect of Small-molecule GSK3 Antagonist on Differentiation of Rat Dental Pulp Cells into Odontoblasts
}

\author{
YOSHIKO MASUDA ${ }^{1}$, HIROSHI SAKAGAMI ${ }^{2}$, SATOSHI YOKOSE ${ }^{3}$ and NOBUYUKI UDAGAWA ${ }^{4}$ \\ ${ }^{1}$ Department of Operative Dentistry, Matsumoto Dental University, Nagano, Japan; \\ ${ }^{2}$ Meikai University Research Institute of Odontology (M-RIO), \\ Meikai University School of Dentistry, Saitama, Japan; \\ ${ }^{3}$ Division of Endodontics and Operative Dentistry, Meikai University School of Dentistry, Saitama, Japan; \\ ${ }^{4}$ Department of Biochemistry, Matsumoto Dental University, Nagano, Japan
}

\begin{abstract}
Background: It has been reported that glycogen synthase kinase 3 (GSK3) antagonist promoted the reparative formation of dentin. The aim of the present study was to evaluate whether treatment schedule of Tidegrusib ${ }^{\circledR}$ (TG), a small-molecule GSK3 antagonist, affected in vitro differentiation of dental pulp cells toward odontoblast-like cells. Materials and Methods: Pulp cells isolated from rat incisors were repeatedly exposed to TG for the first $6 \mathrm{~h}$ (intermittent exposure) or the full $48 \mathrm{~h}$ (continuous exposure) of each 48-h incubation cycle. Histological analysis of alkaline phosphatase and von Kossa staining were performed. The expression of dentin sialophosphoprotein (Dspp) and osteocalcin (Ocn) mRNA were examined by real-time polymerase chain reaction. Western blotting assays were used to monitor the expression of $\beta$-catenin and its phosphorylated form. Results: When pulp cells were intermittently exposed to $T G$ for only the first 6 h of each incubation cycle, pulp cells differentiated into odontoblast-like cells, characterized by an increase in alkaline phosphatase activity, nodule formation, and mRNA expression of Dspp. and Ocn; this did not occur under the continuous exposure. Phosphorylation of $\beta$-catenin was enhanced by continuous exposure to TG compared with intermittent exposure. Conclusion: These results suggest that the TG-induced odontoblast-like cell differentiation reflects in vivo reparative dentin formation and depends on the exposure time.
\end{abstract}

This article is freely accessible online.

Correspondence to: Yoshiko Murakami-Masuda, DDS, Ph.D., Department of Operative Dentistry, Endodontology and Periodontology, Matsumoto Dental University, 1780 Gobara Hirooka, Shiojiri, Nagano 399-0781, Japan. Tel/Fax: +81 263512282, e-mail: yoshiko.masuda@mdu.ac.jp

Key Words: GSK3 antagonist, pulp, Wnt/ $\beta$-catenin signal.
Dental pulp is a connective tissue that contains odontoblasts. The pulp responds to external stimuli, such as cavity preparation, excess tooth wear, restorative materials and caries (1). It produces two forms of tertiary dentin called reactionary dentin and reparative dentin $(2,3)$. Reactionary dentin is formed by primary odontoblasts located at the periphery of the pulp chamber, while reparative dentin is formed by the second generation of odontoblast-like cells that originate from mesenchymal stem cells in the pulp (4).

It is conceivable that signaling molecules expressed by pulp cells play an important role in the healing of pulp during dental repair (5). WNT/ $\beta$-catenin signaling regulates dentin secretion by odontoblasts by directly affecting the formation of reparative dentin (6). Glycogen synthase kinase 3 (GSK3) is a key cytoplasmic component of $\mathrm{WNT} / \beta$ catenin signal transduction, and phosphorylates $\beta$-catenin and axin, leading to their ubiquitination and degradation in the absence of WNT ligand/receptor binding (7). GSK3 is well known to play important roles in restoring the functional integrity of teeth, preserving their vitality and protecting the pulp from further damage.

Direct pulp capping is performed on teeth that have exposed pulp caused by caries, caries excavation, or traumatic injuries. Calcium hydroxide paste and mineral trioxide aggregate are commonly used capping materials (8) that induce reparative dentin formation by their stimuli. It has recently been reported that the small-molecule GSK3 antagonist, Tidegrusib ${ }^{\circledR}$ (also known as NP-12 or NP031112) (TG), promoted reparative dentin formation and restoration in vivo (7). Although TG is a bio-degradable organic material, it induced higher reparative dentin formation compared with non-degradable mineral trioxide aggregate cement that was previously considered the most effective pulp-capping agent (9). Furthermore, TG is under study in clinical trials for treating neurological disorders such as Alzheimer's disease $(10,11)$.

It has been reported that the organic matrix of dentin is deposited at a rate of $4 \mu \mathrm{m} /$ day and mineralized in a 12-hour 
cycle (12). The effect of parathyroid hormone (PTH) on osteoblast differentiation and signal transduction systems has been reported to be considerably variable, depending on the exposure time in vitro (13). Short exposure (6 h) of osteoblastic cells to PTH resulted in several-fold increase in the expression of mRNA for alkaline phosphatase (ALP) and osteocalcin (13). In the present study, we tested the possibility that the intermittent stimulation of rat pulp cells induces differentiation towards odontoblast-like cells and reparative dentin formation more efficiently than does continuous stimulation.

\section{Materials and Methods}

Animals. Wistar strain 5-week-old littermate male rats (Saitama Experimental Animals Supply Co., Ltd., Sugito, Saitama, Japan) (chosen due to easy manipulation of anesthetization and removal of mandibles and pulp) were used to prepare pulp cells, according to the methods established by Yokose et al. (14), after approval by the intramural Animal Care and Use Committee (no. A1927).

Cell culture. Pulp cells were detached from the dish by trypsinization and inoculated onto 6-well plates (Falcon Labware, Corning, NY, USA) at a density of $10^{4}$ cells $/ \mathrm{cm}^{2}$. Cells at the second passage were used for the experiments. They were cultured in $\alpha$-modified Eagle's minimum essential medium-containing $10 \%$ heat-inactivated calf serum (Thermo Fisher Scientific K.K., Tokyo, Japan), $300 \mathrm{mg} / \mathrm{ml} \beta$-glycerophosphate (FUJIFILM Wako Pure Chem. Co., Osaka, Japan), $50 \mathrm{mg} / \mathrm{ml}$ ascorbic acid (FUJIFILM Wako Pure Chem. Co.), and antibiotics (100 mg/ml of penicillin G and $100 \mathrm{IU} / \mathrm{ml}$ of streptomycin) (Thermo Fisher Scientific K.K.).

Experimental protocol for TG exposure. Pulp cells, inoculated at a density of $10^{4}$ cells $/ \mathrm{cm}^{2}$, were cultured for $16 \mathrm{~h}$ to allow complete attachment. They were then divided into three groups and treated as follows: (i) Intermittent exposure: cells were exposed to TG (Monmouth Junction, NJ, USA) $(50 \mathrm{nM})$ for the first $6 \mathrm{~h}$ of each 48-h incubation cycle, and then cultured in the absence of TG for the remainder of the cycle; (ii) continuous exposure: cells were continuously exposed to TG, with a change of culture medium every $48 \mathrm{~h}$; and (iii) control cells were not exposed at all to TG throughout the experimental period. The 48-h culture cycle was repeated eight times, and then the cells were fixed and stained for ALP on day 20.

Histochemistry. The cells were fixed with $10 \%$ neutral buffered formalin, and ALP activity was determined histochemically, using naphthol AS-MX phosphate (substrate) (Sigma-Aldrich Inc. St. Louis, MO, USA), and Fast Red Violet LB Salt (coupler) (SigmaAldrich Inc.), as described previously (15). Mineralized nodules were detected by von Kossa's staining and quantified by counting the number of black and brown nodules in each well of 6-well plates $(n=4)$. The mean \pm SEM were calculated. Statistical treatment was performed with the Mann-Whitney $U$-test. A value of $p<0.05$ was considered statistically significant.

Real-time reverse transcription polymerase chain reaction (RT-PCR). Total RNA was extracted from the pulp cells grown in 6-well plates on days 10 and 20 using RNAiso Plus (Takara Bio Inc., Tokyo, Japan). cDNA was synthesized from total RNA (1 mg) using an RTPCR kit (Takara Bio Inc.) and random primers. Subsequently, 100

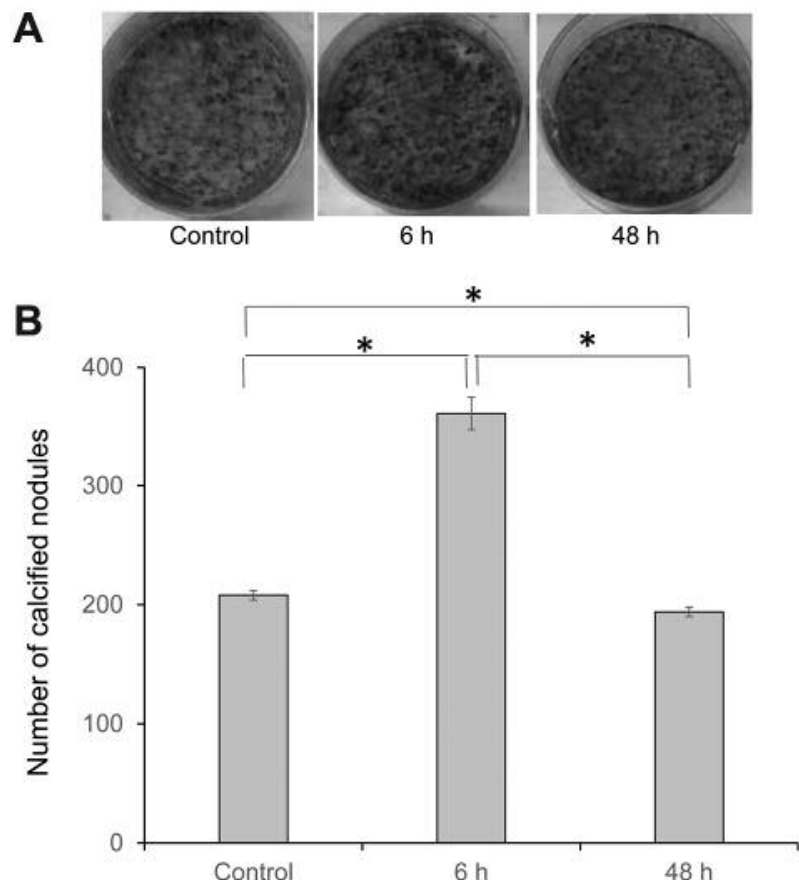

Figure 1. Stimulated formation of calcified nodules in pulp cells by intermittent exposure to Tidegrusib ${ }^{\circledR}(T G)$. A: Pulp cells were exposed to $T G(50 \mathrm{nM})$ for the first $6 \mathrm{~h}$ of each 48-h incubation cycle (intermittent exposure group), or exposed continuously from day 1 to day 17 (the day of termination of the eighth treatment cycle), 3 days before cell harvest) (continuous exposure group), or not exposed for 20 days (control group). Medium changes were performed every $48 \mathrm{~h}$. B: Quantification of the number of calcified nodules from groups as shown in A. Data are the mean $\pm S D$ of four cultures. *Statistically significant at $p<0.05$.

ng cDNA was used as a template for the real-time PCR. Primers were used at $5 \mathrm{mM}$ with $12.5 \mu \mathrm{l} \mathrm{SYBR}$ Green Premix (Takara Biomedical) in a final volume of $25 \mu \mathrm{l}$. SYBR Green PCR amplification and real-time fluorescence detection were performed using a Smart Cycler II System (Takara Biomedical). Denaturation was performed at $95^{\circ} \mathrm{C}$ for $10 \mathrm{~s}$, followed by annealing/extension $(45$ cycles at $95^{\circ} \mathrm{C}$ for $5 \mathrm{~s}$ and $60^{\circ} \mathrm{C}$ for $20 \mathrm{~s}$ ). The sequences of primers (Takara Biomedicals) were: Forward: 5'-CTCAGTTAGTGC CGCTGGAGA-3', reverse: 5'-GAATCGTCGTTAGTGGCGTTG-3' for rat $D s p p$; forward: 5'-AGACTCCGGCGCTACCTCAA-3' and reverse: 5'-CGTCCTGGAAGCCAATGTG-3' for rat Ocn; and forward: 5'-TGACAGGATGCAGAAGGAGGA-3' and reverse: 5'TAGAGCCACCAATCCACACA-3' for rat $\beta$-actin (Actb). Measurements were undertaken during the extension step at $60^{\circ} \mathrm{C}$ in each cycle, and the threshold cycle was calculated by the secondderivative method as described previously (14). The target geneexpression level was normalized to that of Actb in each sample. After amplification, the melting curve of PCR products was analyzed to differentiate between specific and non-specific PCR products.

Western blot analysis. The cells after the first cycle of exposure to TG were washed with PBS, lysed with RIPA solution (Nacalai Tesque, Kyoto, Japan) and processed for western blot analysis, as described previously (16). The intensity of protein expression was 

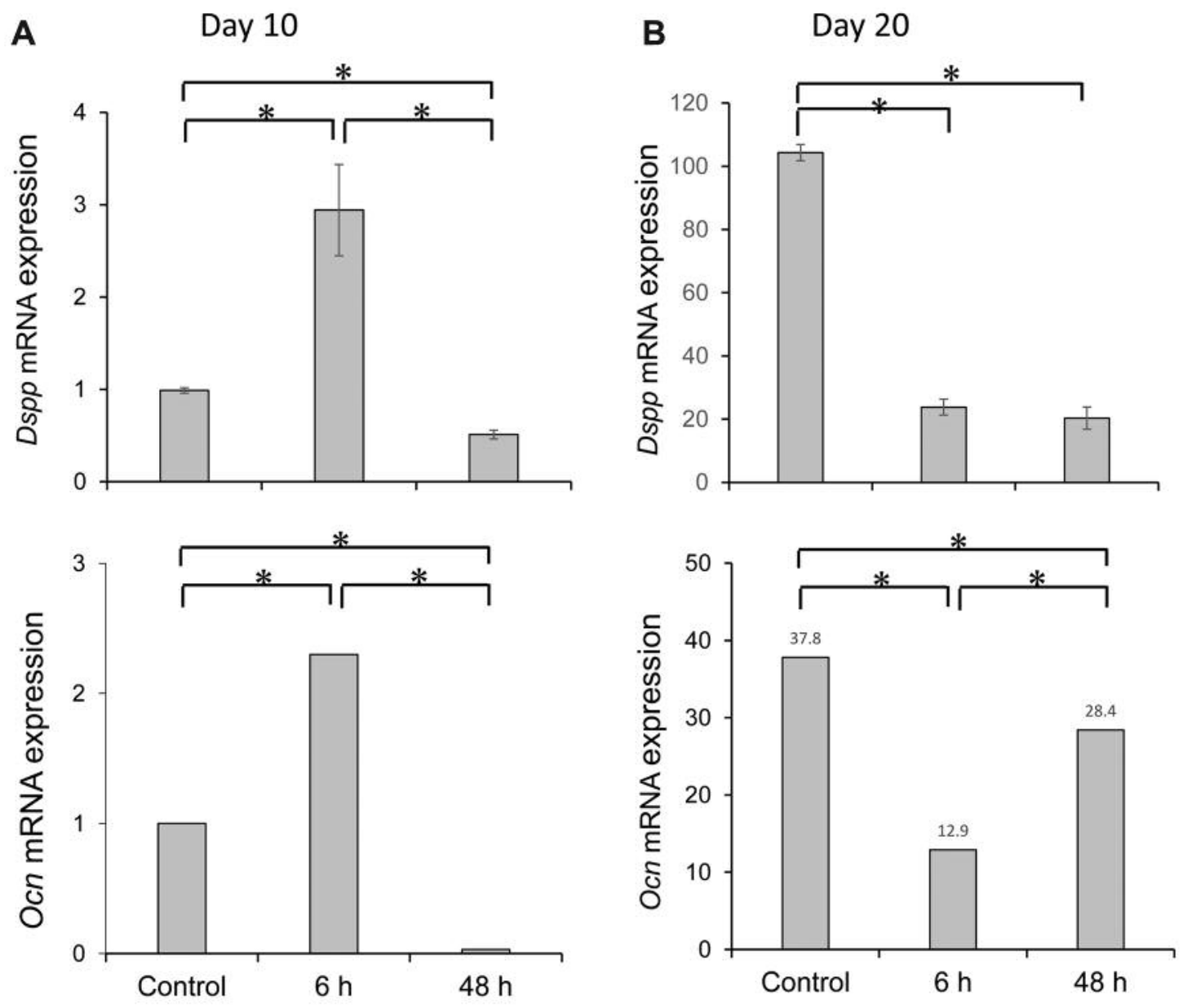

Figure 2. Effects of intermittent and continuous exposure to Tidegrusib ${ }^{\circledR}(T G)$ on the expression of dentin sialophosphoprotein (Dspp) and osteocalcin (Ocn) mRNA. Pulp cells were exposed to TG (50 nM) for 10 (A) or 20 (B) days intermittently for $6 \mathrm{~h}$ or continuously for 48 h, or were not exposed to $T G$ (control), and then Dspp mRNA expression (upper panels) Ocn mRNA expression (lower panels) were quantified by real-time polymerase chain reaction. Each value represents the mean $\pm S D$ of three independent experiments. Mann-Whitney U-test: *Statistically significant at $p<0.05$.

quantified by ImageJ (NIH, Bethesda, MD, USA). As primary antibodies, antibodies against $\beta$-catenin (E247; Abcam, Cambridge, UK), phosphorylated $\beta$-catenin (Phospho-Ser33; Signalway Antibody LLC, College Park, MD, USA) and glyceraldehyde 3phosphate dehydrogenase (GAPDH; Cell Signaling Technology, Inc., Danvers, MA, USA) were used. As a secondary antibody, antirabbit IgG (Cell Signaling Technology, Inc.) antibody conjugated with horseradish peroxidase was used. As a positive control, cells that were continuously exposed to $\mathrm{LiCl}(10 \mathrm{mM})$ for $48 \mathrm{~h}$ in the first cycle were used (17).

\section{Results}

Stimulated formation of calcified nodules by intermittent exposure to $T G$. During incubation for 20 days in $\alpha$-MEM supplemented with $\beta$-glycerophosphate and ascorbic acid, pulp cells spontaneously differentiated into odontoblast-like cells characterized by numerous ALP-positive calcified nodules (Figure 1A). The number of nodules increased in the 6-h TG exposure group (Figure 1A). On the other hand, when pulp cells were treated with TG continuously, the numbers of nodules were slightly but significantly reduced (Figure 1A).

When the number of calcified nodules was quantified, the 6-h intermittent exposure group produced a significantly $(p<0.05)$ higher number of calcified nodues compared with the control and cells exposed continuously to TG (Figure 1B). On the other hand, continuous exposure induced only a slight but significant decrease in the number of nodules compared with the control.

Transient increase of Dspp. and Ocn mRNA expression by intermittent exposure to TG. Real-time PCR analysis demonstrated that intermittent exposure to TG resulted in a 
A

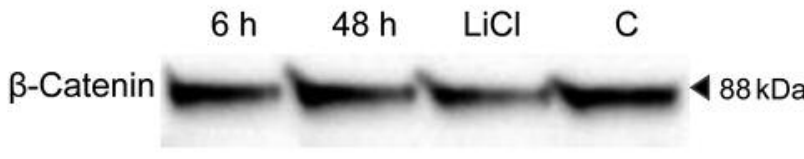

GAPDH

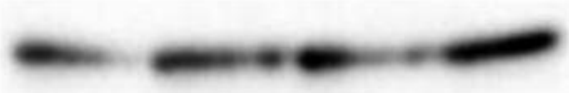

B-Catenin

GAPDH

1.55

1.15

0.93

1.00

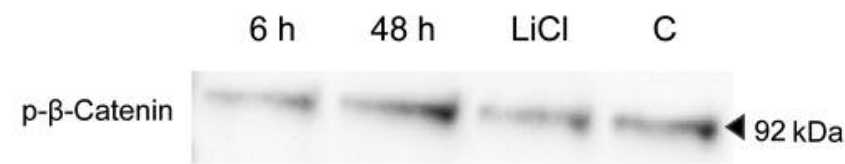

GAPDH

$p-\beta$-Catenin

GAPDH

$0.75 \quad 1.08$

0.69

1.00

Figure 3. Western blot analysis of the expression of $\beta$-catenin and its phosphorylated form after one cycle of exposure for 6 or 48 h to Tidegrusib ${ }^{\circledR}$ $(50 \mathrm{nM})$. Glyceraldehyde-3-phosphate dehydrogenase (GAPDH) served as a loading control. LiCl: Cells were continuously exposed to $10 \mathrm{mM}$ LiCl as a positive control; $C$ : control, cells were incubated without TG. The expression of $\beta$-catenin and its phosphorylated form relative to that of GAPDH was quantified by ImageJ (NIH), and then expressed as a ratio to that of the control.

significant $(p<0.05)$ increase in Dspp and Ocn mRNA on day 10 (2.9 and 2.3-fold increase as compared with control, respectively; Figure 2A) but in a reduction on day 20 (Figure $2 \mathrm{~B})$. On the other hand, continuous exposure to TG did not induce such increases in Dspp and Ocn mRNA on days 10 and 20 but led to a reduction in expression compared with the control (Figure 2B).

Effect on $\beta$-catenin and its phosphorylated form. Western blot analysis demonstrated that after one 48 -h cycle, $\beta$-catenin expression was higher in the 6-h exposure than the 48-h exposure and exposure to $\mathrm{LiCl}$ (used as a positive control) (Figure 3); expression in the control was almost the same as that of the 48-h exposure (Figure 3A). Phosphorylation of $\beta$ catenin was higher with 48 -h exposure than in the 6-h exposure, $\mathrm{LiCl}$ exposure and control (Figure 3B). This indicates higher degradation of phosphorylated $\beta$-catenin with 48-h exposure, that may have reduced its nuclear transport, and the transcription of genes involved in calcification, leading to a decrease of odontoblastic differentiation.

\section{Discussion}

The present study demonstrated that short intermittent $(6 \mathrm{~h})$ exposure to TG (50 $\mathrm{nM})$ induced odontoblastic differentiation. This conclusion is based on our following findings. (i) Sixhour intermittent exposure to TG significantly increased the formation of calcified nodules on day 20 , while continuous 48-h exposure inhibited the formation of the nodules compared with 6-h exposure and the control (Figure 1). (ii) Expression of both Dspp and Ocn mRNA were increased by 6-h exposure compared with 48-h exposure and the control on day 10 (Figure 2). (iii) Expression of $\beta$-catenin and its phosphorylation at Ser33 were enhanced by continuous exposure but not intermittent exposure after $48 \mathrm{~h}$ (Figure 3).
It has previously reported that short-term PTH treatment (5 to $30 \mathrm{~min}$ ) stimulated cell proliferation of osteoblasts more efficiently than long-term treatment (18), possibly due to the selective regulation by cyclic AMP/protein kinase A. The present study demonstrated that continuous exposure to TG induced the phosphorylation of $\beta$-catenin. This suggests that phosphorylated $\beta$-catenin may be degraded in the proteasome (17); without phosphorylation, $\beta$-catenin should be in a monomeric form for translocation to the nucleus to initiate gene transcription (18).

Kadokura et al. reported that phosphorylation of $\beta$-catenin was inhibited by $\mathrm{LiCl}$ in cultured pulp cells (19), consistent with the present finding that phosphorylation of $\beta$-catenin was inhibited by continuous exposure to $\mathrm{LiCl}$, as well as by 6-h exposure to TG after the first cycle (Figure 3).

The WNT signaling pathway is one of the important molecular cascades regulating cell fate. This pathway not only plays a crucial role in growth and development but also in the maintenance of the mature skeleton and response to conditions of loading (20). For maintenance of function, $\mathrm{WNT} / \beta$-catenin signaling may accelerate gene transcription suppressed by the antagonists of WNT receptors including sclerostin, dickkopf-1, -2, and -3 , and secreted frizzledrelated protein 1 protein $(21,22)$. Gene expression is regulated by the balance between accelerator and suppressor to maintain homeostasis. The $6 \mathrm{~h}$-exposure to TG may have accelerated $\beta$-catenin accumulation in the cytoplasm without switching on the suppressor. On the other hand, continuous 48-h exposure may have accelerated the $\beta$-catenin accumulation in the cytoplasm with switching-on of the suppressor, thus leading to the suppression of differentiation of odontoblasts-like cells. Further studies are needed to make clear the mechanism of reparative dentin formation.

In conclusion, the present study suggests that TG has diverse effects on odontoblast-like cell differentiation 
depending on the exposure time in vitro and reflect part of the in vivo action in reparative dentin formation.

\section{Conflicts of Interest}

The Authors wish to confirm that there are no known conflicts of interest associated with this publication and there has been no significant financial support for this work that could have influenced its outcome.

\section{Authors' Contributions}

Y.M. performed all experiments and wrote the article. H.S., S.Y. and N.U. supported the experiments and edited the article.

\section{Acknowledgements}

This study was supported in part by a Grant-In-Aid for Scientific Research (17K11713) from the Ministry of Education, Science, and Culture of Japan and by Miyata Research Fund A from Meikai University School of Dentistry.

\section{References}

1 Tziafas D, Smith AJ and Lesot H: Designing new treatment strategies in vital pulp therapy. J Dent 28: 77-92, 2000. PMID: 10666965. DOI: 10.1016/s0300-5712(99)00047-0

2 Goldberg M and Smith AJ: Cells and extracellular matrices of dentine and pulp: a biological basis for repair and tissue engineering. Crit Rev Oral Biol Med 15: 13-27, 2004. PMID: 14761897. DOI: $10.1177 / 154411130401500103$

3 Hargreaves KM and Berman LH: Cohen's Pathways of the Pulp, Tenth Edition. St. Louis: Mosby Elsevier, pp. 560, 2011.

4 Babb R, Chandrasekaran D, Neves VCM and Sharpe PT: Axin2expressing cells differentiate into reparative odontoblasts via autocrine $\mathrm{Wnt} / \beta$-catenin signaling in response to tooth damage. Sci Rep 7: 3102, 2017. DOI: 10.1038/s41598-017-03145-6

5 Smith $\mathrm{AJ}$ and Lesot $\mathrm{H}$ : Induction and regulation of crown dentineogenesis: Embryonic events as a template for dental tissue repair? Crit Rev Oral Biol Med 12: 425-437, 2001. PMID: 12002824. DOI: $10.1177 / 10454411010120050501$

6 Yuan Z, Xue Y, Teresita B and Jill AH: A correlation between $\mathrm{Wnt} / \beta$-catenin signaling and the rate of dentin secretion. J Endod 45(11): 1357-1364.e1, 2019. PMID: 31522810. DOI: 10.1016/ j.joen.2019.07.014

7 Neves VCM, Babb R, Chandrasekaran D and Sharpe PT: Promotion of natural tooth repair by small molecule GSK3 antagonists. Sci Rep 7: 39654, 2017. DOI: 10.1038/srep39654

8 Hargreaves KM and Berman LH: Cohen's Pathways of the Pulp, Tenth Edition. St. Louis: Mosby Elsevier, pp. 483, 2011.

9 Aguilar $\mathrm{P}$ and Linsuwanont P: Vital pulp therapy in vital permanent teeth with cariously exposed pulp: A systematic review, J Endod 37(5): 581-587, 2011. PMID: 21496652. DOI: 10.1016/j.joen.2010.12.004

10 Tolosa E, Litvan I, Höglinger GU, Burn D, Lees A, Andrés MV, Gómez-Carrillo B, León T and Del Ser T: A phase 2 trial of the GSK3 inhibitor tideglusib in progressive supranuclear palsy. Mov Disord 29(4): 470-478, 2014. DOI: $10.1002 / \mathrm{mds} .25824$
11 Lovestone S, Boada M, Dubois B, Hüll M, Rinne JO, Huppertz HJ, Calero M, Andrés MV, Gómez-Carrillo B, León T and Del Ser T: A phase II trial of tidegrusib in Alzheimer's disease. J Alzheimers Dis 45: 75-88, 2015. PMID: 25537011. DOI: 10.3233/JAD-141959

12 Nanci A: Ten Cate's Oral Histology: Development, Structure, and Function, Ninth Edition. St. Louis: Mosby Elsevier, pp. 173, 2018.

13 Ishizuya T, Yokose S, Hori M, Noda T, Suda T, Yoshiki S and Yamaguchi A: Parathyroid hormone exerts disparate effects on osteoblast differentiation depending on exposure time in rat osteoblastic cells. J Clin Invest 99: 2961-2970, 1997. PMID: 9185520. DOI: 10.1172/JCI119491

14 Yokose S, Kadokura H, Tajima Y, Fujieda K, Katayama I, Matsuoka T and Katayama T: Establishment and characterization of a culture system for enzymatically released rat dental pulp cells. Calcif Tissue Int 66: 139-144, 2000. DOI: 10.1007/ s002230010028

15 Katagiri T, Yamaguchi A, Komaki M, Abe E, Takahashi N, Ikeda T, Rosen V, Wozney JM, Fujisawa-Sehara A and Suda T: Bone morphogenetic protein-2 converts the differentiation pathway of C2C12 myoblasts into the osteoblast lineage. J Cell Biol 127: 1755-1766, 1994. PMID: 7798324. DOI: 10.1083/jcb.127.6.1755

16 Suzuki R, Matsushima Y, Okudaira N, Sakagami H and Shirataki Y: Cytotoxic components against human oral squamous cell carcinoma isolated from Andrographs paniculate. Anticancer Res 36: 5931-5935, 2016. PMID: 27793918. DOI: 10.21873/anticanres.11180

17 Liu C, Li Y, Semenov M, Han C, Baeg GH, Tan Y, Zhang Z and He X: Control of $\beta$-catenin phosphorylation/degradation by a dual-kinase mechanism. Cell 108: 837-847, 2002. PMID: 11955436. DOI: 10.1016/s0092-8674(02)00685-2

18 Scutt A, Duvos C, Lauber J and Mayer H: Time-dependent effects of parathyroid hormone and prostaglandin $\mathrm{E}_{2}$ on DNA synthesis by periosteal cells from embryonic chick calvaria. Calcif Tissue Int 55: 208-215, 1994. PMID: 7987735. DOI: $10.1007 / \mathrm{bf00425877}$

19 Kadokura H, Yamazaki T, Wada Y, Kikui T, Nishimura T, Hirose $\mathrm{K}$, Amano Y and Yokose S: Effects of the inhibition of $\beta$-catenin phosphorylation by $\mathrm{LiCl}$ on differentiation and ectodin expression in rat odontoblast-like cells. Japan J Conserv Dent 56: 231-237, 2013.

20 Duan $\mathrm{P}$ and Bonewald LF: The role of the Wnt $/ \beta$-catenin signaling pathway in formation and maintenance of bone and teeth. Int J Biochem Cell Biol 77: 23-29, 2016. PMID: 27210503. DOI: 10.1016/j.biocel.2016.05.015

21 Burgers TA and Williams BO: Regulation of Wnt/ $\beta$-catenin signaling within and from osteocytes. Bone 54: 244-249, 2013. PMID: 23470835. DOI: 10.1016/j.bone.2013.02.022

22 Canalis E: Wnt signaling in osteoporosis: Mechanism and novel therapeutic approaches. Nat Rev Endocrinol 9: 575-583, 2013. PMID: 23938284. DOI: 10.1038/nrendo.2013.154

Received February 19, 2020

Revised March 17, 2020

Accepted March 18, 2020 